\title{
Evaluation of Geographic Indices Describing Health Care Utilization
}

\author{
Agnus M. Kim 1, Jong Heon Park², Sungchan Kang ${ }^{3}$, Yoon Kim ${ }^{1,3}$ \\ ${ }^{1}$ Department of Health Policy and Management, Seoul National University College of Medicine, Seoul, ${ }^{2}$ Big Data Steering Department, National \\ Health Insurance Service, Wonju; ${ }^{3}$ Institute of Health Policy and Management, Seoul National University Medical Research Center, Seoul, Korea
}

Objectives: The accurate measurement of geographic patterns of health care utilization is a prerequisite for the study of geographic variations in health care utilization. While several measures have been developed to measure how accurately geographic units reflect the health care utilization patterns of residents, they have been only applied to hospitalization and need further evaluation. This study aimed to evaluate geographic indices describing health care utilization.

Methods: We measured the utilization rate and four health care utilization indices (localization index, outflow index, inflow index, and net patient flow) for eight major procedures (coronary artery bypass graft surgery, percutaneous transluminal coronary angioplasty, surgery after hip fracture, knee replacement surgery, caesarean sections, hysterectomy, computed tomography scans, and magnetic resonance imaging scans) according to three levels of geographic units in Korea. Data were obtained from the National Health Insurance database in Korea. We evaluated the associations among the health care utilization indices and the utilization rates.

Results: In higher-level geographic units, the localization index tended to be high, while the inflow index and outflow index were lower. The indices showed different patterns depending on the procedure. A strong negative correlation between the localization index and the outflow index was observed for all procedures. Net patient flow showed a moderate positive correlation with the localization index and the inflow index.

Conclusions: Health care utilization indices can be used as a proxy to describe the utilization pattern of a procedure in a geographic unit.

Key words: Small-area analysis, Health care, Utilization, Variation, Insurance, Korea

\section{INTRODUCTION}

Geographic variation in health care utilization has been a major issue in health care policy and research for decades. If an optimal amount of health care utilization exists for a popu-

Received: October 14, 2016, Accepted: December 19, 2016

Corresponding author: Yoon Kim, MD, PhD

103 Daehak-ro, Jongno-gu, Seoul 03080, Korea

Tel: +82-2-740-8363, Fax: +82-2-743-2009

E-mail: yoonkim@snu.ac.kr

This is an Open Access article distributed under the terms of the Creative Commons Attribution Non-Commercial License (http://creativecommons.org/licenses/bync/4.0// which permits unrestricted non-commercial use, distribution, and reproduction in any medium, provided the original work is properly cited. lation in a geographically defined area, deviation from that optimal amount can be indicative of a problem in terms of both the efficiency and effectiveness of health care. Studies of geographic variation have been performed to address that concern.

Defining geographic units of analysis is crucial for analyzing and interpreting geographic variation in health care utilization [1]. Since one of the ultimate purposes of geographic variation studies is to identify supplier factors in variation, geographic units must describe the health care utilization of the residents accurately. A geographic unit of analysis, ideally, should be self-sufficient in health care utilization, so that geographic health care utilization can be correctly attributed to the influence of the area's health care supply [2]. Geographic variation 
analysis based on units that do not describe geographic patterns of health care utilization accurately may erroneously attribute health care utilization to health care suppliers [3]. While organizing geographic units of analysis has received considerable attention as an important issue in geographic variation studies [1,2], the evaluation of geographic units with regard to their relevance as units of analysis has received less attention.

However, evaluating geographic units is as important as defining new geographic units for the following reasons. First, the majority of variation studies are based on administrative units. Administrative units are not organized for the purpose of variation analysis and are likely to be inconsistent with actual health care utilization. Additionally, even small areas organized for the purpose of variation analysis are not consistent with geographic patterns of health care utilization [4-6]. Therefore, geographic units of analysis should be evaluated for their accuracy in describing geographic health care utilization. Second, comparing degrees of variation is an inevitable part of making judgments about the degree of variation. To make a fair comparison, differences in the degree to which each geographic unit reflects actual health care utilization should be considered. Therefore, the comparison of variation across different geographic units should be accompanied by an evaluation of the degree to which those geographic units accurately describe actual health care utilization.

Although several measures have been developed to measure how accurately geographic units reflect the health care utilization of the residents, they have only been tentatively applied to hospitalization in a small number of studies [4-6] and require further evaluation. This study aimed to evaluate the indices describing geographic health care utilization by investigating how these indices reflected utilization patterns and the extent to which these indices were associated with health care utilization for major health care procedures.

\section{METHODS}

\section{Data}

This study used data from the National Health Insurance (NHI) database for the 2013 period. The NHI database contains data for the entire Korean population, including NHI beneficiaries ( $97 \%$ of the country's population) and medical aid beneficiaries (3\% of the total population) [7]. We acquired information on patient demographics, the geographic location of hospitals, diagnosis codes, and procedure codes from the NHI da- tabase. Diagnoses were classified by the Korean Standard Classification of Diseases codes, and procedures were classified by the Electronic Database Interchange codes.

Utilization rates of the following eight procedures were calculated: coronary artery bypass graft (CABG) surgery, percutaneous transluminal coronary angioplasty (PTCA), surgery after hip fracture, knee replacement surgery, hysterectomy, caesarean sections, computed tomography (CT) scans, and magnetic resonance imaging (MRI) scans. These procedures were chosen both for their importance in terms of cost, volume, and policy relevance [8] and for their diverse characteristics in terms of urgency, frequency, and severity (Table 1). Utilization rates of the eight procedures were calculated based on the patient's place of residence. Rates were age-adjusted and sex-adjusted to the Korean resident population of 2013. The caesarean section rate was standardized to the number of live births in Korea in 2013 according to the age of the mother. Rates were calculated per 100000 population aged 15 and over. For the CABG and PTCA rates, the population age was 20 and over. The rate of caesarean sections was calculated per 1000 live births.

We used three kinds of administrative units as geographic units of analysis: districts, cities, and provinces. Administrative units in Korea are categorized into provincial-level units (special metropolitan city, metropolitan city, special self-governing city, province, and special self-governing province) and municipal-level units (city, county, and district) [3]. A district is a subunit of a city. However, due to the large variation in population size among cities, a district of a large city can have a larger population than a small city. Therefore, the municipal level can be applied in two ways: city/county, with strict application of

Table 1. Characteristics of the 8 major procedures

\begin{tabular}{lccc}
\hline & Urgency & Frequency & Severity \\
\hline CABG & 0 & & 0 \\
PTCA & 0 & $\Delta$ & 0 \\
Surgery after hip fracture & 0 & $\Delta$ & \\
Knee replacement surgery & & $\Delta$ & \\
Hysterectomy & $\Delta$ & $\Delta$ & $\Delta$ \\
Caesarean sections & & 0 & \\
CT scans & & 0 & \\
MRI scans & & & \\
\hline Eren
\end{tabular}

Frequency was based on the annual rate of the procedures in 2013 [3]. The circle represents a high degree and the triangle represents a moderate degree of each characteristic. The urgency and severity of caesarean sections are denoted with triangles, considering both the emergent nature of the caesarean section and the high proportion of elective caesarean sections in Korea. CABG, coronary artery bypass graft; PTCA, percutaneous transluminal coronary angioplasty; CT, computed tomography; MRI, magnetic resonance imaging. 
the hierarchy of the administrative units, or city/county/district, considering the population size of the unit. This study applied both methods of classification. To represent each administrative unit level, we adopted the name of the predominant administrative unit at each level. Therefore, for the municipal level, this study used 163 cities and 251 districts, and for provincial level, this study used 16 provinces as administrative units. The average population of each unit was 203751 (districts, 313751 (cities), and 3196341 (provinces). The range of population size for each unit was 10524 to 668425 (districts), 10524 to 10143645 (cities), and 593806 to 12234630 (provinces).

\section{Statistical Analysis}

This study examined four metrics for assessing geographic health care utilization: the localization index, the inflow index, the outflow index, and net patient flow. The localization index, inflow index, and net patient flow were originally developed as measures for hospitalization $[4]$ and discharges $[5,6]$. The outflow index was newly designed in this study. These metrics are presented in Figure 1 [5].

We defined the localization index as the fraction obtained by dividing the number of instances of a procedure performed in an area and received by the residents of the area (A in Figure 1) by the total number of procedures done on the residents in that area ( $A$ and $C$ in Figure 1). The localization index can be understood as the extent to which residents' demands for health care are satisfied in their area of residence or as the resi-

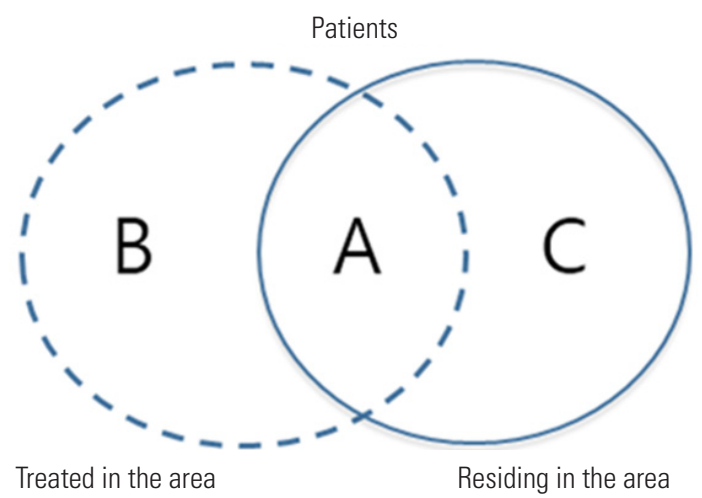

Localization index $=A /(A+C)$

Inflow index $=B /(A+B)$

Outflow index $=C /(A+B+C)$

Net patient flow $=\mathrm{B} / \mathrm{C}$

Figure 1. Geographic indices describing health care utilization. Modified from Kilaru et al. Med Care 2015;53(6):510-516 [5]. dents' tendency to receive health care in their own area [5].

The inflow index was defined as the fraction obtained by dividing the number of instances of a procedure performed on non-residents ( $B$ in Figure 1 ) by the total number of procedures performed within an area ( $A$ and $B$ in Figure 1 ). It is indicative of the degree to which patients are drawn from other areas [5] or a surplus in health care supply that may facilitate care for non-residents.

We invented the outflow index to estimate the residents' unmet needs for health care services. With the goal of distinguishing the outflow of patients due to a lack of resources from outflow due to patients' own preferences, we calculated the outflow index as the fraction of the number of instances of a procedure performed on residents outside of their geographic area ( $C$ in Figure 1$)$ divided by the sum of the total number of instances of that procedure performed on the residents ( $A$ and $C$ in Figure 1 ) and the number of procedures performed on non-residents within a given area (B in Figure 1). The number of procedures received by non-residents in the denominator was included to take into account the reserve in the health care resources of an area. If this additional component becomes larger, the value of the outflow index decreases. As it contains the number of procedure performed on nonresidents in its denominator, the outflow index is not complementary to the localization index. We expected the outflow index to have more explanatory power for the distribution of utilization across geographic units than the localization index.

Net patient flow is the ratio of the number of instances of a procedure performed on non-residents within an area ( $B$ in Figure 1) to the number of instances of a procedure performed on residents outside of that area ( $B$ in Figure 1 ) [5]. A value over 1 indicates that patient import is predominant over patient export, and a value under 1 denotes the preponderance of patient export over patient import. As discussed below, net patient flow can be used as an index indicative of the overconcentration of procedures performed in an area.

We calculated the four utilization indices for the eight procedures according to three levels of geographic units, and investigated the associations between the indices and the utilization rates.

The study was carried out using SAS version 9.4 (SAS Institute Inc., Cary, NC, USA), SPSS version 23 (IBM Corp., Armonk, NY, USA), and R statistical software (R Foundation for Statistical Computing, Vienna, Austria). 


\section{RESULTS}

The localization index, inflow index, outflow index, and patient net flow were calculated for the eight major procedures according to the three levels of Korean administrative units (Table 2). In the case of the localization index, higher-level geographic units tended to show higher values. The localization index for all geographic units was the lowest for CABG. The localization index was higher for surgery after hip fracture and caesarean sections.
The inflow index tended to be lower in higher-level geographic units (provinces), but the difference among geographic units was not as distinct as it was for the localization index. Knee replacement surgery showed overall high values of the inflow index.

The outflow index was the lowest in higher-level geographic units (provinces). The outflow index was the highest for CABG, and tended to be lower for surgery after hip fracture, caesarean sections, and CT scans than for other procedures.

In the case of net patient flow, we used the median value for

Table 2. Values of health care utilization indices for the 8 major procedures according to 3 levels of administrative units in Korea

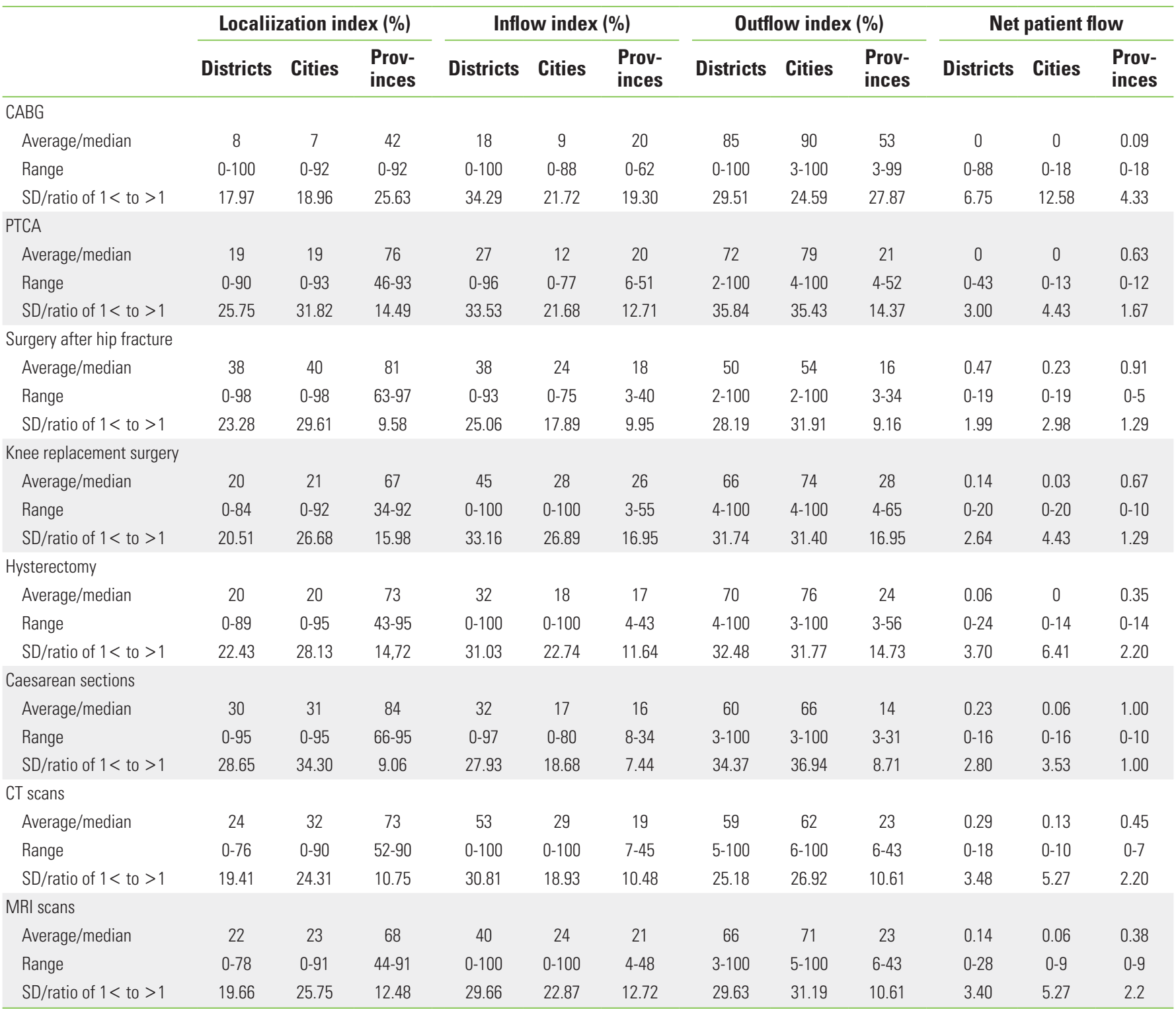

We calculated the average and SD for the localization index, inflow index, and outflow index; For net patient flow, we calculated the median and the ratio of the number of units where the net patient flow was $<1$ to the number of units where the net patient flow was $>1$.

CABG, coronary artery bypass graft; PTCA, percutaneous transluminal coronary angioplasty; CT, computed tomography; MRI, magnetic resonance imaging; SD, standard deviation. 


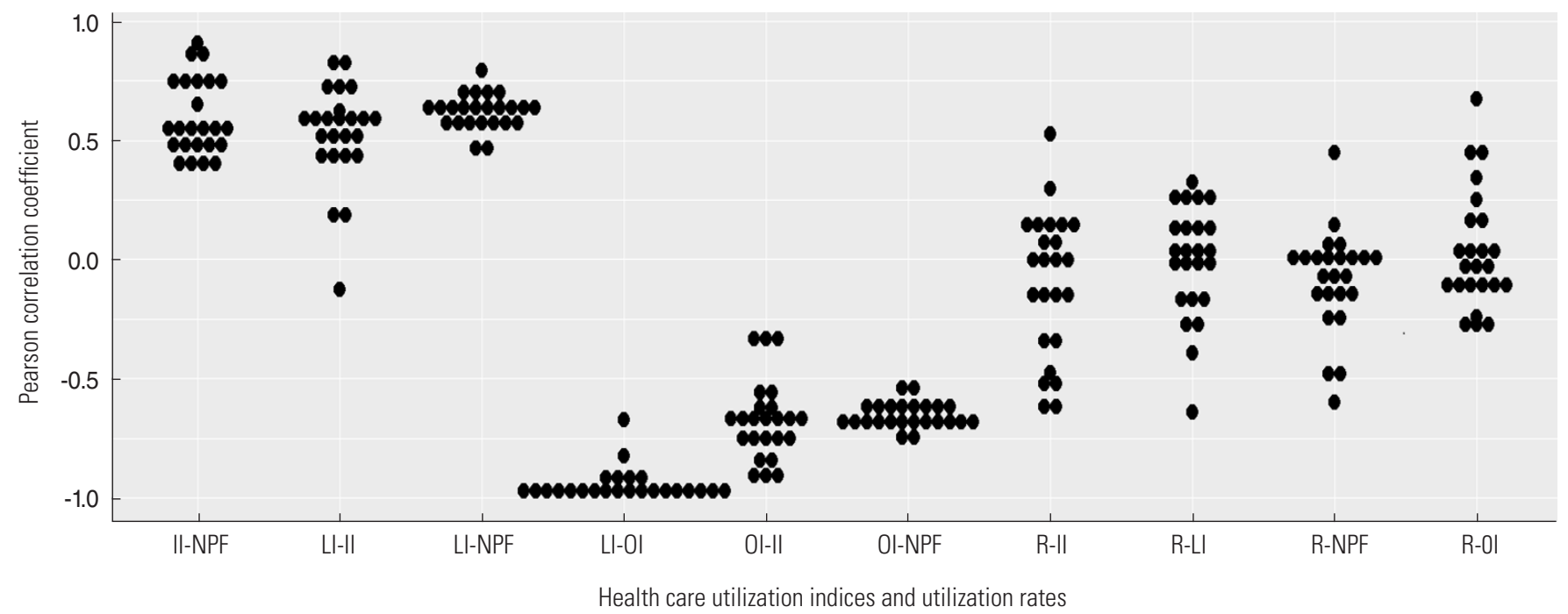

Figure 2. Relationships among the health care utilization indices and the utilization rates. LI, localization index; II, inflow index; Ol, outflow index; NPF, net patient flow. R: procedure utilization rate.

comparison considering the distribution of net patient flow which was right-skewed. CABG showed the lowest value of this parameter in all geographic units. Surgery after hip fracture, caesarean sections, and CT scans tended to have higher values of net patient flow.

We assessed the relationship among health care utilization indices (Figure 2). A strong negative correlation was identified between the localization index and outflow index for all procedures. A moderate positive correlation between the inflow index and net patient flow was found. The localization index and net patient flow also showed a moderate positive correlation.

We assessed the relationships among utilization rates and health care utilization indices (Figure 2, Table 3). The rate of knee replacement surgery showed a negative correlation with the localization index, inflow index, and net patient flow, and a positive correlation with the outflow index in all geographic units. For CT scans, the inflow index and net patient flow showed a negative correlation with the utilization rate, and the outflow index showed a positive correlation with the utilization rate in provinces. For surgery after hip fracture and PTCA, the utilization rate showed a positive correlation with the localization index and a negative correlation with the outflow index in districts and cities.

\section{DISCUSSION}

In this study, we investigated the relationships among health care utilization indices and the utilization rates of eight major procedures to evaluate how the indices related to one another and how the indices explained utilization patterns. We confirmed that the associations among health care utilization indices and utilization rates differed according to the characteristics of the procedures and across geographic units.

The localization index was higher for higher-level geographic units. That indicates that interprovincial movement for health care utilization was less frequent than intercity or interdistrict movement. However, even on the level of provinces, values of the localization index differed markedly among procedures. The lowest value of the localization index was for $C A B G$, indicating that the need of residents for this procedure was met the least among the procedures that we studied. As shown in the total number of procedures performed in 2013 [3], CABG is an infrequently performed procedure. Its relative rarity may have influenced the quality or quantity of the supply of available health care facilities, resulting in more frequent interprovincial movement for health care utilization. In addition, the cardiologist performing the index treatment and the hospital where the patients are treated are known to strongly influence patients' decision-making on coronary revascularization [9], and patient's decisions tend to reflect their own preferences [10]. Such characteristics, which allow some discretion in decision-making, may have also contributed to the high interregional movement of the patients. Surgery after hip fracture showed a high localization index, which reflects the characteristics of the procedure. One important characteristic is the relative urgency of the procedure, and the other is that lit- 
Table 3. Relationships among the utilization rates and the health care utilization indices

\begin{tabular}{|c|c|c|c|c|c|c|c|c|}
\hline & & & & & & & & \\
\hline & PCC & $p$-value & PCC & $p$-value & PCC & $p$-value & PCC & $p$-value \\
\hline CABG & & & & & & & & \\
\hline Districts & 0.052 & 0.41 & 0.101 & 0.11 & -0.079 & 0.21 & 0.014 & 0.83 \\
\hline Cities & 0.039 & 0.62 & 0.122 & 0.12 & -0.043 & 0.59 & 0.060 & 0.45 \\
\hline Provinces & 0.106 & 0.69 & 0.524 & 0.04 & -0.141 & 0.60 & 0.450 & 0.08 \\
\hline PTCA & & & & & & & & \\
\hline Districts & 0.287 & $<0.001$ & 0.164 & 0.009 & -0.244 & $<0.001$ & 0.060 & 0.34 \\
\hline Cities & 0.322 & $<0.001$ & 0.162 & 0.04 & -0.305 & $<0.001$ & 0.146 & 0.06 \\
\hline Provinces & -0.046 & 0.86 & -0.128 & 0.64 & 0.052 & 0.85 & -0.169 & 0.53 \\
\hline Surgery after & & & & & & & & \\
\hline Districts & 0.248 & $<0.001$ & 0.122 & 0.05 & -0.241 & $<0.001$ & 0.031 & 0.62 \\
\hline Cities & 0.247 & 0.001 & 0.125 & 0.11 & -0.249 & 0.001 & 0.016 & 0.84 \\
\hline Provinces & -0.041 & 0.88 & 0.294 & 0.27 & -0.011 & 0.97 & 0.010 & 0.97 \\
\hline Knee replace & & & & & & & & \\
\hline Districts & -0.253 & $<0.001$ & -0.550 & $<0.001$ & 0.435 & $<0.001$ & -0.236 & $<0.001$ \\
\hline Cities & -0.292 & $<0.001$ & -0.370 & $<0.001$ & 0.343 & $<0.001$ & -0.123 & 0.12 \\
\hline Provinces & -0.643 & 0.007 & -0.588 & 0.02 & 0.671 & 0.004 & -0.511 & 0.04 \\
\hline Hysterectomy & & & & & & & & \\
\hline Districts & 0.151 & 0.02 & 0.004 & 0.95 & -0.093 & 0.14 & 0.003 & 0.97 \\
\hline Cities & 0.138 & 0.08 & -0.021 & 0.79 & -0.117 & 0.14 & 0.014 & 0.86 \\
\hline Provinces & 0.010 & 0.97 & -0.477 & 0.06 & 0.041 & 0.88 & -0.258 & 0.33 \\
\hline Caesarean se & & & & & & & & \\
\hline Districts & 0.018 & 0.77 & -0.160 & 0.01 & 0.051 & 0.42 & -0.055 & 0.39 \\
\hline Cities & 0.040 & 0.61 & 0.045 & 0.57 & -0.049 & 0.54 & -0.086 & 0.27 \\
\hline Provinces & -0.150 & 0.58 & -0.490 & 0.05 & 0.171 & 0.53 & 0.454 & 0.08 \\
\hline CT scans & & & & & & & & \\
\hline Districts & -0.143 & 0.02 & 0.016 & 0.80 & 0.156 & 0.01 & -0.151 & 0.02 \\
\hline Cities & 0.006 & 0.94 & -0.174 & 0.03 & 0.017 & 0.83 & -0.157 & 0.04 \\
\hline Provinces & -0.394 & 0.13 & -0.647 & 0.007 & 0.459 & 0.07 & -0.599 & 0.01 \\
\hline MRI scans & & & & & & & & \\
\hline Districts & 0.230 & $<0.001$ & -0.128 & 0.04 & -0.106 & 0.09 & 0.022 & 0.73 \\
\hline Cities & 0.158 & 0.04 & -0.020 & 0.80 & -0.137 & 0.08 & -0.015 & 0.84 \\
\hline Provinces & -0.189 & 0.48 & -0.316 & 0.23 & 0.249 & 0.35 & -0.071 & 0.79 \\
\hline
\end{tabular}

LI, localization index; II, inflow index; OI, outflow index; NPF, net patient flow; PCC, Pearson correlation coefficient; CABG, coronary artery bypass graft; PTCA percutaneous transluminal coronary angioplasty; CT, computed tomography; $\mathrm{MRI}$, magnetic resonance imaging.

tle discretion exists regarding treatment options. As a result of these characteristics, surgery after hip fracture has been used as a reference procedure in geographic variation studies [8]. Caesarean sections likewise showed a high localization index. The caesarean section rate is Korea is among the highest in the world [11], and geographic variation in the caesarean section rate in Korea has been found to be low compared with other countries [3]. It can be postulated that the regional supply for caesarean sections is sufficient to maintain a high localization index with little variation, and the tendency to choose a caesarean section is quite strong in Korea despite the discretionary nature of many caesarean sections $[12,13]$.

The inflow index tended to decrease in higher-level geographic units. This corresponds to the findings for the localization index to some extent. As the degree of localization increases in geographic units, patient export to other areas decreases. Thus, from the perspective of the other areas, inflow of patients may decrease. Additionally, as we considered the inflow index to indicate the degree to which patients were drawn from other areas, lower values of the inflow index in 
higher-level geographic units may indicate that the degree to which an area attracted patients from other areas decreased in higher-level geographic units. The lower value of the inflow index in higher-level geographic units also indicates that interareal movement was likely to be less frequent among higher-level geographic units than among lower-level geographic units. Infrequent movement among higher-level geographic units may be partially due to the higher cost incurred by longdistance travel. In addition, this result reflects the health care delivery system in Korea, which is mainly based on the municipal level for the most common and rudimentary aspects of health care.

The outflow index was lower in higher-level geographic units. This decrease was more prominent than for the inflow index. As will be discussed further below, the sharper decrease of the outflow index in higher-level geographic units can be explained by its high correlation with the localization index. The outflow index values for surgery after hip fracture and for caesarean sections, which showed higher localization index values, were lower than for other procedures. CABG, which showed the highest value for the localization index, had the lowest outflow index value.

As net patient flow approaches zero, it indicates a preponderance of patient export over patient import. In the case of $C A B G$, the median value of the net patient flow index was near zero in all geographic units, which means that the number of geographic units in which patient export predominated was far greater than the number of geographic units in which patient import predominated. Therefore, we can see that CABG was performed more intensively in a small number of areas, which means that the degree of concentration was greater than for other procedures. The net patient flow was comparatively high for caesarean sections and surgery after hip fracture, and CT scans, indicating that the procedures were likely to be performed relatively evenly across geographic units.

The strongest correlation among the health care utilization indices was found between the localization index and the outflow index. The deviation from -1 (a perfect negative correlation) may be due to the impact of the non-residents treated in an area on the number of procedures performed on residents. The correlation between the localization index and the outflow index was less than -0.9 in most cases. Since the value of the inflow index ranged from 16 to $53 \%$, we could suppose that the inflow of patients was not negligible. However, as indicated by the value of net patient flow, patient outflow was predominant over patient inflow in a large proportion of geographic units. Therefore, the strong negative correlation between the localization index and outflow index likely reflected the predominance of patient outflow over patient inflow, with patient import having a minimal impact on the denominator of the outflow index.

The correlation between the outflow index and the inflow index was strongly negative, except in the case of surgery after hip fracture and CT scans. The relatively weak correlation observed for surgery after hip fracture and CT scans may be explained by the fact that for these procedures, it is less necessary to search to find better care. Concerning surgery after hip fracture, it has been suggested that interventions be performed within approximately 24 hours of the injury because earlier surgery is more advantageous in terms of outcomes and complications [14-17]. This characteristic of urgency may have reduced the tendency for patients to travel to another area for treatment. In the case of CT scans, there is less room on the part of the patients to seek better quality, which may be related with less patient movement among areas. The positive correlation between the inflow index and net patient flow reflects the fact that net patient flow increased with patient import. Additionally, the positive correlation between the localization index and net patient flow suggests that patient import was positively related with the degree of localization.

The relationships among utilization rates and health care utilization indices varied across procedures. In knee replacement surgery, the utilization rate generally showed significant correlations with the health care utilization indices. The negative correlation between the utilization rate and localization index in knee replacement surgery suggests that more knee replacements were performed on patients who sought care outside their own area of residence. This fact indicates that in the case of knee replacement surgery, patients who sought care outside their own residence were more likely to undergo surgery. The positive correlation between the utilization rate and the outflow index in knee replacement surgery can be explained in the same way. It is known that evidence-based indications have not been established for knee replacement surgery and that the treatment decision for knee replacement surgery is dependent on the patient's opinion [18]. These characteristics of knee replacement surgery make patients seeking care outside their own area more likely to receive treatment that may be unnecessary.

The inflow index and net patient flow showed negative cor- 
relations with the rate of knee replacement surgery. The negative correlation between the inflow index and utilization rate suggests that the residents in areas where many non-residents came to undergo procedures tended to receive fewer procedures. This explanation is consistent with the interpretation of the negative association between the localization index and the utilization rate. While patients who sought care outside their own area of residence tended to receive more procedures, patients who stayed in their own area of residence tended to have fewer procedures. Therefore, patients who lived in an area where patient outflow predominated over patient inflow tended to undergo more procedures, as shown by the negative correlation between the utilization rate and net patient flow in knee replacement surgery.

The relationships between the utilization rate and the four health care utilization indices in CT scans are similar to those observed for knee replacement surgery. However, in the case of CT scans, the correlation appeared stronger at the provincial level. This can be explained in two ways. First, this may merely be the effect of the modifiable areal unit problem, which refers to variation caused by the different ways in which data can be aggregated to form areal units [19]. Second, it may be due to the traits of the procedure. CT scans, a diagnostic procedure, are more likely to be performed in conjunction with the main medical treatment, except in cases of health examinations among the healthy population. Patients are less likely to travel to a different area just to receive a CT scan; it is therefore more likely that patients travel to a different area to undergo specific medical procedures that require the use of CT scans. It may be postulated that patients who desire to undergo certain procedures in different provinces are more prone to undergo CT scans. In that case, we can suppose that these procedures may be complicated or expensive, since they induce patients to travel across provinces.

In surgery after hip fracture and PTCA, a positive correlation between the utilization rate and the localization index and a negative correlation between the utilization rate relation between the utilization rate and the localization index and a negative correlation between the utilization rate and the outflow index were observed at the municipal level. Under the premise that overuse is less likely for these procedures, the positive correlation between the utilization rate and the localization index suggests that the residents of an area with a high localization index were more likely to receive the treatment they needed. The negative correlation between the utilization rate and the outflow index can be explained in a similar way. The more patient outflow occurred, the less likely it was that residents received treatment.

This study has several limitations. First, while the number of most of the procedures in this study can be considered to reflect all procedures performed in Korea, we were unable to determine how many MRI scans were not covered by the $\mathrm{NHI}$. Therefore, the statistical findings presented in this study on MRI scans are likely to differ from the actual utilization rate in Korea. Second, the variation in population size among Korean administrative units is greater than that observed in other countries [3]. As large variation in population sizes is known to cause extra-variation, variation in the population size may have influenced the measurement of the utilization rate and indices. Third, a causal relationship is different from simple associations. To interpret associations and to develop causal interpretations, we need more information on the factors that may affect health care utilization. With information on the socioeconomic status of each geographic unit and the distribution of health care facilities within geographic units, we would be able to interpret these associations more accurately and to elucidate the causal relationships underlying our findings.

To the best of our knowledge, this is the first study to attempt to examine health care utilization indices by characterizing the relationships among the indices and the health care utilization rates. By applying the health care utilization indices to major medical procedures, instead of hospitalization or discharge, we have broadened the scope of application of these health care utilization indices. For a more detailed explanation of the relationships among the health care utilization indices, we need to investigate the specific factors that may affect such relationships.

Accurate measurements of geographic patterns of health care utilization are a prerequisite for the study of geographic variation in health care utilization. We evaluated four health care utilization indices describing the geographic patterns of health care utilization for eight major procedures according to three levels of geographic units in Korea. In higher-level geographic units, the localization index was high, while the inflow index and outflow index were relatively low. Additionally, the indices showed different patterns for different procedures. A strong negative correlation between the localization index and the outflow index was observed in all eight procedures. Net patient flow showed a moderate positive correlation with the localization index and the inflow index. We found that 
health care utilization indices could be used as a proxy to describe the utilization pattern of specific procedures in various geographic units.

\section{ACKNOWLEDGEMENTS}

This article is based on a thesis submitted by the first author to the Seoul National University College of Medicine in partial fulfillment for the Master of Science in Medicine. This study was supported by the National Health Insurance Service.

\section{CONFLICT OF INTEREST}

The authors have no conflicts of interest associated with the material presented in this paper.

\section{ORCID}

Angus M. Kim http://orcid.org/0000-0002-6616-2534

Sungchan Kang http://orcid.org/0000-0002-4876-3205

Yoon Kim http://orcid.org/0000-0001-7257-1679

\section{REFERENCES}

1. Thygesen LC, Baixauli-Pérez C, Librero-López J, Martínez-Lizaga N, Ridao-López M, Bernal-Delgado E, et al. Comparing variation across European countries: building geographical areas to provide sounder estimates. Eur J Public Health 2015;25 Suppl 1:8-14.

2. Volinn E, Diehr P, Ciol MA, Loeser JD. Why does geographic variation in health care practices matter? (And seven questions to ask in evaluating studies on geographic variation). Spine (Phila Pa 1976) 1994;19(18 Suppl):2092S-2100S.

3. Kim AM, Park JH, Kang S, Hwang K, Lee T, Kim Y. The effect of geographic units of analysis on measuring geographic variation in medical services utilization. J Prev Med Public Health 2016;49(4):230-239.

4. Dartmouth Medical School, Center for the Evaluative Clinical Sciences. The Dartmouth atlas of health care. Chicago: American Hospital Pub.; 1996, p. 15, 22.

5. Kilaru AS, Wiebe DJ, Karp DN, Love J, Kallan MJ, Carr BG. Do hospital service areas and hospital referral regions define discrete health care populations? Med Care 2015;53(6):510-516.

6. Klauss G, Staub L, Widmer M, Busato A. Hospital service areas
-- a new tool for health care planning in Switzerland. BMC Health Serv Res 2005;5:33.

7. Song YJ. The South Korean health care system. Japan Med Assoc J 2009;52(3):206-209.

8. Organization for Economic Cooperation and Development. Focus on health: geographic variations in health care; 2014 [cited 2016 Aug 20]. Available from: https://www.oecd.org/ els/health-systems/FOCUS-on-Geographic-Variations-inHealth-Care.pdf.

9. Tu JV, Ko DT, Guo H, Richards JA, Walton N, Natarajan MK, et al. Determinants of variations in coronary revascularization practices. CMAJ 2012;184(2):179-186.

10. Brownlee S, Wennberg JE, Barry MJ, Fisher ES, Goodman DC, Bynum JP. Improving patient decision-making in health care: a 2011 Dartmouth atlas report highlighting Minnesota; 2011 [cited 2017 Jan 11]. Available from: http://www.dartmouthatlas.org/downloads/reports/Decision_making_report_022411. pdf.

11. Organization for Economic Cooperation and Development. Health at a glance 2015: OECD indicators [cited 2017 Jan 11]. Available from: http://www.oecd.org/health/health-systems/ health-at-a-glance-19991312.htm.

12. Wagner M. Choosing caesarean section. Lancet 2000;356 (9242):1677-1680.

13. Penna $L$, Arulkumaran S. Caesarean section for non-medical reasons. Int J Gynaecol Obstet 2003;82(3):399-409.

14. Simunovic N, Devereaux PJ, Bhandari M. Surgery for hip fractures: does surgical delay affect outcomes? Indian J Orthop 2011;45(1):27-32.

15. Moran CG, Wenn RT, Sikand M, Taylor AM. Early mortality after hip fracture: is delay before surgery important? J Bone Joint Surg Am 2005;87(3):483-489.

16. Bottle A, Aylin P. Mortality associated with delay in operation after hip fracture: observational study. BMJ 2006;332(7547): 947-951.

17. Turner P, Cocks J, Cade R, Ewing H, Collopy B, Thompson G. Fractured neck of the femur (DRG 210/211): prospective outcome study. Aust N Z J Surg 1997;67(2-3):126-130.

18. Hawker GA. Who, when, and why total joint replacement surgery? The patient's perspective. Curr Opin Rheumatol 2006; 18(5):526-530.

19. Openshaw S, Taylor PJ. A million or so correlation coefficients: three experiments on the modifiable areal unit problem. Stat Appl Spat Sci 1999;21:127-144. 